\section{O controle do câncer do colo do útero: desafios para implementação de ações programáticas no Amazonas, Brasil}

\section{Cervical cancer prevention: challenges facing the implementation of program actions in Amazonas, Brazil}

Dina Albuquerque Duarte Corrêa 1

Wilza Vieira Villela 2

1,2 Programa de Mestrado em Promoção de Saúde. Pró-reitoria de Pesquisa. Universidade de Franca. Av. Dr. Armando Salles Oliveira, 201. Parque Universitário. Franca, SP, Brasil. CEP: 14.404-600 E-mail: dinacorrea@bol.com.br

\begin{abstract}
The purpose of this study is to present a number of challenges facing the implementation of program actions regarding the control of cervical cancer in the State of Amazonas, Brazil. Reports of the Viva Mulher program in Amazonas on Papanicolaou screening tests and the quality of cervical samples and mortality rates from this disease taken from DATASUS were analyzed for the 2001 to 2005 period. The results show no increase in the population covered by the Papanicolaou screening test in this period, although there was a small improvement in the quality of cervical samples. However, mortality rates for cervical cancer are increasing in this reason, and are higher than the rates for Brazil as a whole and for the North region of the country. Analysis of these results suggests the need for local program managers, to make efforts to build the capacity of health-care professionals and service managers, with a view to increasing Papanicolaou screening test coverage for high-risk women.
\end{abstract}

Key words Uterine Cervical Neoplasms, Health Programs and Plans, Health Promotion, Vaginal Smears

\section{Resumo}

O objetivo deste artigo é demonstrar alguns desafios para a implementação de ações programáticas visando ao controle do câncer do colo do útero no Estado do Amazonas, Brasil. Foram analisados os relatórios do Programa Viva Mulher do Amazonas referentes à coleta e qualidade das laminas e os dados de mortalidade por esta causa registrados no DATASUS para os periodos 2001 a 2005. Os resultados apresentam que no período não houve o aumento na cobertura da coleta de exames de Papanicolaou, embora tenha havido uma discreta melhora na qualidade das lâminas; as taxas de mortalidade por câncer de colo de útero estão aumentando, e situam-se em patamares acima dos encontrados no país e na região norte. A análise desses resultados sugere a necessidade de haver um esforço, por parte da gestão do Programa no Amazonas, na capacitação de profissionais e dos gerentes dos serviços, visando aumentar a captação das mulheres sob maior risco.

Palavras-chave Câncer do colo do útero, Planos e Programas de Saúde, Promoção da saúde, Esfregaço vaginal 


\section{Introdução}

O câncer do colo de útero (CCU) representa um importante problema de saúde pública em países em desenvolvimento, chegando a ser em algumas regiões o tipo de câncer mais comum na população feminina. ${ }^{1}$ A incidência desta doença depende da exposição a fatores de risco e da falta de efetividade de programas de rastreamento, ${ }^{2}$ para os quais o exame Papanicolaou tem se mostrado útil em reduzir a incidência e mortalidade por essa neoplasia. ${ }^{3,4}$

Maior benefício é alcançado quando o exame é realizado em mulheres acima de 35 anos, ${ }^{5}$ priorizando-se, assim, o rastreamento na faixa etária entre 35 e 59 anos, pois nessa idade encontram-se as mulheres mais acometidas pela doença. 6

Uma forma organizada de controle do câncer do colo uterino é necessária para que possa ser observada redução em suas taxas de incidência e mortalidade, o que implica na estruturação de uma política de prevenção.

Apesar de o exame Papanicolaou ser uma modalidade de screening capaz de reduzir as taxas de incidência de câncer do colo do útero, a cobertura desse ainda é baixa na população feminina brasileira. ${ }^{7}$

Neste artigo é feita uma análise das ações programáticas para o controle do câncer do colo uterino no Estado do Amazonas, visando identificar os principais desafios para que esta atividade apresente maior efetividade.

Na primeira parte é apresentado o Programa Nacional de Prevenção do Câncer do Colo do Útero, que se inseriu nas contas do Ministério da Saúde a partir de 1996, década esta em que o controle da doença passou a fazer parte da agenda da política brasileira de saúde. 8

$\mathrm{Na}$ segunda parte avalia-se a maneira como o sistema público de saúde do Estado vem trabalhando para o controle dessa neoplasia, procurando compreender as ações de prevenção após a introdução do Programa Viva Mulher no Amazonas, bem como o impacto na população feminina e a qualidade dos resultados citológicos. Para tanto, além da consulta aos relatórios do programa no Estado, foram coletadas e analisadas informações a partir de registros do Departamento de Informação e Informática do Sistema Único de Saúde (DATASUS).

Vale ressaltar que, no Estado do Amazonas, o combate ao câncer uterino foi estabelecido recentemente, acompanhando o Programa de Prevenção Nacional (relatório das atividades da Gerência dos Programas de Prevenção e Controle do Câncer no
Amazonas, GEPROG, 2001). São registradas elevadas taxas de incidência e mortalidade por essa neoplasia no Estado. ${ }^{9}$ Em revisão bibliográfica exaustiva em base de dados Pubmed, LILACS e SciELO, e nos acervos das universidades locais, verificou-se ainda uma escassez de estudos a este respeito no Estado do Amazonas.

\section{A criação do Programa Nacional de Controle do Câncer do Colo do Útero}

No Brasil, a saúde da mulher foi incorporada às políticas nacionais nas primeiras décadas do século $\mathrm{XX}$, sendo o atendimento limitado ao ciclo gravídico-puerperal. 10 No final dos anos 60 , foi criado o Programa de Saúde Materno Infantil, cujo enfoque estava em prestar assistência aos filhos das mulheres que não tinham acesso aos serviços de saúde, constituindo a atenção pré-natal como única ação de caráter universalista com relação à saúde da mulher. ${ }^{11}$

Com as mudanças na condição social das mulheres, ocorridas ao longo da segunda metade do século XX, as propostas de atenção materno-infantil mostram-se insuficientes. Em 1984 é proposto, pelo Ministério da Saúde, o Programa de Assistência Integral à Saúde da Mulher (PAISM), visando à extensão das ações básicas em saúde da mulher e incluindo, entre outras, atividades preventivas e de diagnóstico do câncer do colo do útero e mamário. A assistência integral envolvia o atendimento de todas as necessidades da população feminina, enfatizando as ações dirigidas ao controle das patologias mais prevalentes, incluindo ações educativas.12,13 Porém, a implementação efetiva do Programa não aconteceu como desejado, ficando as atividades nos serviços de saúde bastante focalizadas nas demandas relativas à contracepção, acompanhamento da gravidez e ao parto. A prevenção e controle do câncer ginecológico continuaram em segundo plano. 10

Em 1998 é instituído o Programa Nacional de Controle do Câncer do Colo do Útero (PNCCCU), considerado como uma estratégia mais adequada para mudar o cenário desse câncer no país. ${ }^{8}$

O PNCCCU foi estruturado com a meta de reduzir a mortalidade e as repercussões físicas, psíquicas e sociais por esse câncer, através da oferta de serviços para prevenção e detecção em estágios iniciais, além de disponibilizar tratamento e reabilitação. $10 \mathrm{O}$ Programa elegeu como método rastreador o exame de Papanicolaou (colpocitologia oncológica) e elegeu a cirurgia de alta freqüência (CAF) como método de tratamento das lesões intraepiteliais de alto grau, priorizando as mulheres com idades 
entre 35 e 49 anos e as que nunca haviam realizado o exame preventivo. 14

Inicialmente, foi elaborado um Projeto Piloto denominado Viva Mulher, implantado no ano de 1997 em apenas seis localidades brasileiras (Curitiba, Brasília, Recife, Rio de Janeiro, Belém e em todo Estado de Sergipe). Com base na experiência do Projeto Piloto Viva Mulher, foi realizada em 1998 a expansão nacional, com inclusão de todos os Estados, abrangendo 97,9\% dos municípios brasileiros. 10

De agosto a setembro de 1998 foi realizada a Primeira Fase de Intensificação do Programa, viabilizada por meio de uma campanha que utilizou os diversos meios de comunicação para mobilização da população. A população alvo eram mulheres de 35 a 49 anos e as que nunca haviam realizado o exame preventivo, e a meta era atingir, no prazo de 60 dias, o teto de quatro milhões de exames, tendo sido realizados 3.177.740 exames citopatológicos. 10

A partir de 1999, foi iniciada a Fase de Consolidação das Ações do Programa na rotina dos serviços. Em 2002, nos meses de março e abril, foi realizada a Segunda Fase de Intensificação do Programa, sendo desenvolvida uma campanha de mídia nacional para mobilização das mulheres alvo. Pretendia-se, nesta fase, atingir a meta de 2.511.581 mulheres. No entanto, o programa superou as expectativas, alcançando um total de 3.856.650 mulheres examinadas. 10

Para efetivação do Programa Nacional de Controle do Câncer do Colo do Útero, dois pontos foram contemplados. O primeiro foi a sensibilização da população feminina para realização do exame, sendo este respondido com divulgação da campanha através dos meios de comunicação escrito, falado e televisionado. O segundo ponto importante para viabilização do programa foi a sensibilização dos profissionais de saúde da rede, realizada por meio de treinamentos dos gerentes municipais do Programa e profissionais de saúde da rede, elaboração de materiais educativos e apresentação da proposta para os recursos humanos envolvidos na atenção ao câncer do colo do útero, com sua justificativa epidemiológica, objetivos, metas e orientações para o seguimento de cada mulher.

\section{Programa Nacional de Controle do Câncer do Colo do Útero no Amazonas}

No Estado do Amazonas, em 1998, houve a implantação do Programa Nacional de Controle do Câncer do Colo do Útero, atingindo os 62 municípios amazonenses, onde se registrou, nesse período, um universo superior a 40.000 mulheres submetidas ao exame citológico de Papanicolaou. É interessante observar que, apesar do Amazonas apresentar uma elevada taxa de incidência de CCU, sendo o terceiro Estado com a maior taxa do país, ${ }^{9}$ até 1999 não se dispunha de dados reais de cobertura do exame citológico no Estado, quando esse era realizado pelo Sistema Único de Saúde em apenas dois municípios, Manaus e Manacapuru, conforme relatório das atividades da Gerência dos Programas de Prevenção e Controle do Câncer no Amazonas - GEPROG em 2001.

Em 1999, deu-se início à estruturação funcional do Programa Viva Mulher nas Unidades Básicas de Saúde do Estado. Desde o início da implantação do programa no Amazonas, a equipe responsável vem utilizando várias estratégias para organizar a rede de serviços. Segundo o relatório das atividades da Gerência dos Programas de Prevenção e Controle do Câncer no Amazonas em 2002, dentre as estratégias utilizadas para efetivar o programa no Estado, podemos citar: capacitação dos profissionais para coleta do exame; distribuição de kits ginecológicos; criação do Cartão de Prevenção da Mulher; distribuição de panfletos e livretos; realização de palestras educativas em escolas; educação durante o comparecimento à Unidade de Saúde; e seguimento dos casos detectados, entre outras medidas.

O estudo dos relatórios das atividades da Gerência dos Programas de Prevenção e Controle do Câncer no Amazonas demonstra que, no ano de 2001, servidores de 46 municípios receberam capacitação gerencial sobre o Programa e profissionais de saúde dos 62 municípios do Estado foram treinados para coleta de material para exame citopatológico. Apesar da continuidade do processo de capacitação dos profissionais, a má qualidade da amostra do exame citopatológico e a pouca informação dos profissionais de saúde sobre o Programa foram algumas questões levantadas pelo diagnóstico situacional do mesmo, apontando, assim, uma possível deficiência na replicação dos treinamentos.

A análise dos dados relativos ao número de exames coletados nas Unidades de Saúde do Estado que foram enviados para pagamento pelo Sistema Único de Saúde (SUS) no período da série histórica, revelou que houve um aumento no número de coletas realizadas de 2001 para 2002, ampliando de 84.178 para 135.35 exames. Entretanto, é importante ressaltar que em 2003 houve uma queda nos números apresentados, sendo registrados 114.157 exames. O aumento do número de exames de 2001 para 2002 poderia ser decorrente de um efeito pós- 
Figura 1

Número de exames citopatológicos coletados. Amazonas, 2001 a 2005.

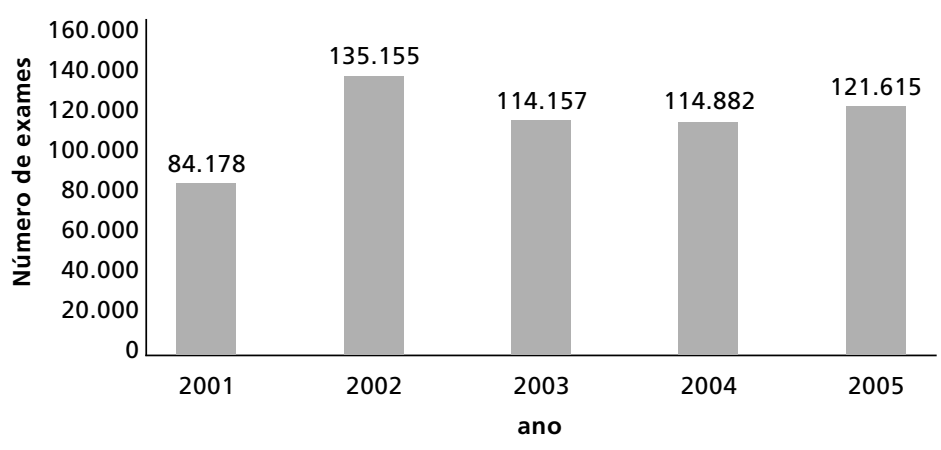

Fonte: Brasil. Ministério da Saúde; SUS (Sistema Único de Saúde), Departamento de Informação e Informática; SISCOLO (Sistema de Informações de Controle do Câncer do Colo do Útero). Informações Estatísticas. [monografia online] 2005.20

campanha, uma vez que em 2002 foi lançada a segunda fase de Intensificação do Programa, enquanto que o declínio do número de exames em 2003 sinaliza uma possível deficiência no processo de continuidade de divulgação e sensibilização para realização do exame.15 (Figura 1)

Com relação ao número de exames coletados nos anos de 2003 para 2004, a produtividade se manteve estável, havendo uma discreta ampliação de 114.157 para 114.882 exames, indicando uma provável saturação na oferta do serviço de saúde, por falta de investimento ou de profissionais capacitados. Em 2005, houve um crescimento no número de coletas realizadas, sendo registrados 121.615 exames. 15

Analisando o número de coletas realizadas na população alvo do Programa Viva Mulher (35 a 49 anos), a partir de dados primários do DATASUS de 2002 a 2005, o percentual de exames realizados nessa faixa etária em relação ao total foi, respectivamente, 39, 27, 27 e 28\%. Esses dados permitem observar que em 2002 o Programa conseguiu atingir um maior percentual de coletas na população alvo, enquanto nos anos seguintes ficou evidente que tal alcance não ocorreu satisfatoriamente, havendo um deslocamento da realização dos exames para um público cuja faixa etária não era o objetivo principal do Programa, de forma que o direcionamento e a divulgação adequada do mesmo ao público alvo possam ser questionados.

No Brasil não é possível saber o número de mulheres examinadas, mas sim a quantidade de exames realizados, dificultando assim calcular a cobertura da população. ${ }^{16}$ A melhor organização da coleta de dados sobre os exames realizados e seu processamento poderia evitar que a duplicidade de coletas interferisse na análise do alcance do programa, bem como facilitaria o ordenamento dos recursos.

Buscando compreender melhor a oferta de exames e sua necessidade, calculou-se a razão entre o número de exames citopatológicos cérvico-vaginais realizados por mulheres de 25 e 59 anos e o número de mulheres nessa faixa etária. No período de 2002 a 2005 , essa razão foi respectivamente de $0,21,0,15,0,15$ e 0,15 exames/mulher/ano, valores esses de tendência estável nos últimos anos e abaixo da razão mínima esperada, de 0,3 , indicando que a oferta do exame Papanicolaou está aquém da necessidade do Estado. 16

Outro aspecto analisado foi a adequabilidade das lâminas. A partir de dados primários do DATASUS, foi feita uma comparação do percentual de lâminas consideradas satisfatórias, satisfatórias mas limitadas e insatisfatória, para o período 2002-2005. A análise revelou percentuais baixos, embora crescentes no final do período, de lâminas com adequabilidade satisfatória; sendo obtidos, respectivamente, percentuais de 26, 25, 33 e 43\% a cada ano. A análise demonstrou, para o mesmo período, a mesma tendência de percentuais baixos de lâminas insatisfatórias, apresentando respectivamente valores de 5 , 5,6 e $3 \%$. Entretanto, os dados revelam percentuais elevados de lâminas satisfatórias mas limitadas, observando-se, de 2002 a 2005, valores de, respectivamente, $69,70,61$ e $54 \%$.

Esses resultados da adequabilidade das lâminas obtidos no período de 2002 a 2005 mostram que os percentuais de lâminas insatisfatórias está dentro do preconizado pela Organização Pan-americana da Saúde (OPAS), ou seja, abaixo que $5 \%$, exceto no ano de 2004, que ficou acima do recomendado. ${ }^{16}$ Apesar disto, observam-se percentuais elevados de lâminas com limitação para leitura. A redução desses percentuais no final do período, que corresponde também ao aumento do percentual de laminas satisfatórias, sugere que o esforço na capacitação dos profissionais realizado no âmbito do Programa viva Mulher foi bem sucedido. No entanto, deve-se ressaltar que o percentual de lâminas satisfatórias ainda é baixo. Esse fato pode ser explicado por técnica inadequada de coleta, resultante de poucos recursos materiais ou humanos capacitados, bem como por condições inerentes às pacientes, como doenças infecciosas cérvico-uterinas, que podem ser algumas vezes o motivo da busca do exame pela mulher desinformada sobre a sua especificidade. 17 
Enfatiza-se, assim, a necessidade de o Programa capacitar os profissionais para coleta de material para exame citopatológico, uma vez que a situação da falta de adequabilidade das lâminas é um dos fatores responsáveis pelos resultados falso-negativos, 18 bem como divulgar informação sobre o exame à população.

Outra questão a ser evidenciada são os dados sobre a mortalidade por câncer do colo do útero, já que a redução do número de mortes causadas por essa neoplasia inclui-se entre os objetivos do Programa.

Ao analisar o coeficiente de mortalidade por câncer do colo uterino no Estado do Amazonas foi identificado coeficientes com valores, respectivamente, de $5,5,6,6,6,4,7,7$ e 8,2 óbitos por 100.000 mulheres 19 (Figura 2). Esses valores apresentaram tendência à ascensão ao longo dos cinco anos, sugerindo que um dos objetivos do Programa, a redução do coeficiente de mortalidade pelo câncer uterino, ainda não foi atingido. Entretanto, a relação entre estas taxas crescentes de óbitos e as ações do Programa, bem como as campanhas de intensificação, devem ser analisadas com cautela nestes anos iniciais de programa, uma vez que, a partir da estimativa de tempo médio de evolução do câncer de colo de mais de 10 anos até o desfecho, pode-se aventar a hipótese de que mulheres que foram a óbito já estivessem com lesões avançadas quando da campanha, não podendo ser beneficiadas pelas ações de detecção e tratamento precoce das lesões. Assim, estudos mais específicos devem ser feitos de modo a estabelecer associações mais precisas entre esse aumento de mortalidade e as ações do Programa.

Procedendo a uma avaliação do comportamento dos coeficientes de mortalidade por câncer do colo do útero no Brasil e na Região Norte, nota-se que em ambos os casos os valores seguem uma tendência estável com um discreto aumento. No Brasil, os coeficientes registrados no período de 2001 a 2004 foram, respectivamente, 4,8,4,6, 4,6 e 4,7 por 100.000 mulheres, e, na Região Norte, 4,9, 5,5, 5,4 e 5,9 por 100.000 mulheres. Comparando esses dados com os encontrados no Estado, observou-se que no Amazonas houve um maior crescimento nos coeficientes, mantendo números sempre acima dos registrados no Brasil e na Região Norte. ${ }^{19}$

Considerando as taxas de mortalidade por câncer uterino padronizadas por idades em alguns países latino-americanos como Argentina, Chile, Costa Rica e México, temos, no ano de 2001, respectivamente, taxas de $4,6,8,6,8,8$ e 11,4 óbitos por 100.000 mulheres. 20 Deste modo, as taxas encontradas no Brasil, na Região Norte e no Estado do
Figura 2

Coeficiente de mortalidade por câncer do colo do útero por 100.000 mulheres. Amazonas, 2001 a 2005.

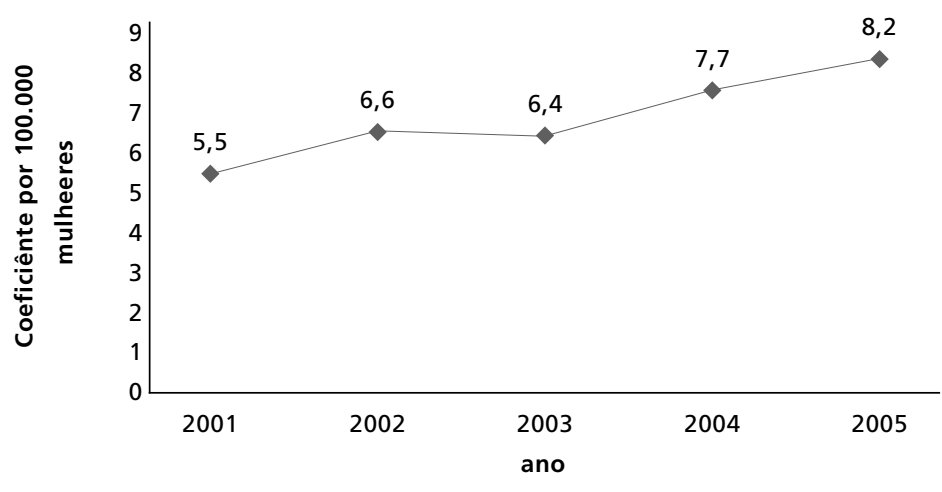

Fonte: Brasil. Ministério da Saúde. SUS (Sistema Único de Saúde), Departamento de Informação e Informática. SIM (Sistema de Informação sobre Mortalidade). Indicadores e dados básicos: Brasil, 2007 [monografia online]. 2007.20

Amazonas, embora diferentes entre si, se apresentam um pouco mais próximas das taxas da Argentina, e abaixo daquelas dos demais países citados.

Esses dados confirmam o fato de que a tendência de mortalidade por câncer uterino no mundo é desigual, sendo determinada pelo modo particular com que diferentes fatores se articulam no determinado contexto, como os hábitos e práticas sexuais da população, a estrutura e organização dos sistemas de saúde e a qualidade dos serviços. No entanto, tanto em países da Europa Ocidental quanto em países da América do Norte as taxas de mortalidade são baixas, sendo, por exemplo, registrada na França e no Canadá em 2002 uma taxa de mortalidade padronizada por idade de, respectivamente, 1,4 e 1,5 por 100.000 mulheres. ${ }^{21}$ Logo, a maior proporção de óbitos por câncer uterino ocorre em mulheres da America Latina, ficando nítida sua associação com a pobreza, o acesso limitado aos serviços de saúde e o baixo nível educacional da população. ${ }^{20,22}$

\section{Considerações finais}

Os dados apresentados mostram que, para o período de 2001 a 2005, apesar de ter ocorrido uma evolução no rastreamento do câncer do colo do útero no Estado do Amazonas, os números estão muito aquém do preconizado. A razão entre o exame citopatológico em mulheres de 25 a 59 anos e a população 
nessa faixa etária ficou abaixo da razão mínima esperada para o Brasil, evidenciando-se a necessidade de aprimoramento das ações de rastreamento de câncer uterino.

Observou-se, ainda, que não ocorreu um aumento significativo do número de exames e que a captação de novas mulheres não está sendo direcionada à população sob maior risco, que se constitui o alvo do programa. Esse fato realça a necessidade do planejamento de ações para alcance das mulheres com maior risco para a doença, a fim de reduzir as taxas de incidência e mortalidade por câncer uterino.

A análise dos dados sobre adequabilidade das lâminas revelou que existe um grande percentual de lâminas com limitação para leitura, podendo indicar baixa capacitação dos profissionais que realizam a coleta do material, bem como recursos materiais inadequados. Frente a essa situação, o treinamento dos profissionais para realização do exame, incluído

\section{Referências}

1. Silva DW, Andrade SM, Soares DA, Turini B, Schneck CA, Lopes MLS. Cobertura e fatores associados com a realização do exame Papanicolaou em município do Sul do Brasil. Rev Bras Ginecol Obstetr. 2006; 28: 24-31.

2. Feitosa TMP, Almeida RT. Perfil de produção do exame citopatológico para controle do câncer do colo do útero em Minas Gerais, Brasil, em 2002. Cad Saúde Pública. 2007; 23: $907-17$

3. Gustafsson L, Pontén J, Zack M, Adami HO. International incidence rates of invasive cervical cancer after introduction of cytological screening. Cancer Causes Control. 1997; 8: 755-63.

4. Cesar JA, Horta BL, Gomes G, Houlthausen RS, Willrich RM, Kaercher A, Iastrenski FM. Fatores associados à não realização de exame citopatológico de colo uterino no extremo sul do Brasil. Cad Saúde Pública. 2003; 19: 136572.

5. Miller AB, Robles SC. Taller centro americano sobre la detección del cáncer de cuello uterino. Bol Sanit Panam. 1996; 121: 589-602.

6. Pinho AA, França Junior I. Prevenção do câncer de colo de útero: um modelo teórico para analisar o acesso e a utilização do teste de Papanicolaou. Rev Bras Saúde Matern Infant. 2003; 3: 95-112.

7. Eluf-Neto J, Nascimento CM. Cervical cancer in Latin America. Semin Oncol. 2001; 28: 188-97.

8. Brasil. Ministério da Saúde. Secretaria Executiva. Controle do câncer do colo do útero. Brasília, DF; 2001.

9. Brasil. Ministério da Saúde. Secretaria de Atenção à Saúde, INCA (Instituto Nacional de Câncer). Coordenação de Prevenção e Vigilância de Câncer. Estimativas 2008: incidência de câncer no Brasil. Rio de Janeiro; 2007. entre os objetivos do Programa, deve ser contínuo, bem como as condições de coleta, transporte e leitura das lâminas devem ser cuidadosamente monitoradas, evitando assim resultados falso-negativo.

Ressalta-se também a importância de analisar as dificuldades encontradas no acesso das mulheres ao exame, vinculadas à estrutura e à organização da assistência em saúde, visando evitar a duplicação desnecessária de coletas e incluir ações na rotina dos serviços que facilitem a captação de adesão das mulheres sob maior risco. Nesta perspectiva, talvez fosse importante implementar ações voltadas para aumentar o conhecimento das mulheres a respeito dos métodos de prevenção do câncer uterino, sensibilizando-as para realização do exame preventivo. ${ }^{23}$

Esses são alguns dos desafios que devem ser enfrentados para tentar reverter o incômodo quadro de aumento de óbito de mulheres pelo câncer de colo uterino no Estado do Amazonas.
10. Brasil. Ministério da Saúde. Secretaria de Assistência à Saúde, INCA (Instituto Nacional de Câncer). Programa Viva Mulher. Câncer do colo do útero: informações técnicogerenciais e ações desenvolvidas. Rio de Janeiro; 2002.

11. Villela WV, Monteiro S. Atenção à saúde das mulheres: historicizando conceitos e práticas. In: Wilza Villela; Simone Monteiro, organizadores. Gênero e saúde: Programa Saúde da Família em questão. São Paulo: Arbeit; 2005. p. 15-31.

12. Brasil. Ministério da Saúde. Secretaria de Atenção à Saúde. Departamento de Ações Programáticas Estratégicas. Política Nacional de Atenção Integral à Saúde da Mulher: princípios e diretrizes. Brasilia, DF; 2004.

13. Osis MJMD. PAISM: um marco na abordagem da saúde reprodutiva no Brasil. Cad Saúde Pública. 1998; 14: 25-32.

14. Roberto Neto A, Ribalta JCL, Focchi J, Baracat EC. Avaliação dos métodos empregados no Programa Nacional de Combate ao Câncer do Colo Uterino do Ministério da Saúde. Rev Bras Ginecol Obstetr. 2001; 23: 209-15.

15. Brasil. Ministério da Saúde; SUS (Sistema Único de Saúde), Departamento de Informação e Informática; SISCOLO (Sistema de Informações de Controle do Câncer do Colo do Útero). Informações estatísticas. [monografia online] 2005. [acesso em: 17 fev. 2008] Disponível em: http://tabnet.datasus.gov.br/cgi/deftohtm.exe?siscolo/DEF/ todos/AMCCOLO.def

16. Brasil. Ministério da Saúde, Secretaria de Atenção à Saúde, INCA (Instituto Nacional de Câncer). Coordenação de Prevenção e Vigilância. A situação do câncer no Brasil. Rio de Janeiro; 2006.

17. Tavares SBN, Amaral RG, Manrique EJC, Sousa NLA, Albuquerque ZBP, Zeferino LC. Controle da qualidade em citopatologia cervical: revisão de literatura. Rev Bras Cancerol. 2007; 53: 355-64. 
18. Souen J, Carvalho JP, Pinotti JA. Oncologia genital feminina. 2. ed. São Paulo: Roca; 2001.

20. Brasil. Ministério da Saúde; SUS (Sistema Único de Saúde), Departamento de Informação e Informática. SIM (Sistema de Informação sobre Mortalidade). Indicadores e dados básicos, Brasil: 2007 [monografia online]. 2007 [acesso em: 17 fev. 2008] Disponível em: http://tabnet. datasus.gov.br/cgi/deftohtm.exe?idb2003/c10.def

20. Lewis, MJ. Análisis de la situación del cáncer cervicouterino en América Latina y el Caribe. Washington, DC: Pan American Health Organization; 2004.

21. WHO (World Health Organization). Summary table by cancer [monograph online]. 2002. [cited 2008 Mar 11] Available from: http://www.dep.iarc.fr/WHO/table2.asp? cancer $=102 \&$ sex $=2 \&$ age_from $=1 \&$ age_to $=18 \&$ period $=200$ $2 \&$ sort $=0 \&$ submit $=$ Execute

Recebido em 3 de julho de 2008

Versão final apresentada em 27 de outubro de 2008

Aprovado em 29 de outubro de 2008
22. Sanjosé S, Bosch FX, Muñoz N, Tafur L, Gili M, Izarzugaza I, Izquierdo A, Navarro C, Vergara A, Muñoz MT, Ascunce N, Shah KV. Socioeconomic differences in cervical cancer: two case-control studies in Colombia and Spain. Am J Public Health. 1996; 86: 1532-8.

23. Roberto Neto A, Ribalta JCL, Focchi J. Baracat EC. Avaliação crítica da estrutura do Programa Nacional de Combate do Câncer de Colo Uterino do Ministério da Saúde. Femina. 2001; 29: 555-60. 